\title{
Macrophage-secreted IL-8 induces epithelial-mesenchymal transition in hepatocellular carcinoma cells by activating the JAK2/STAT3/Snail pathway
}

\author{
XIU-TAO FU ${ }^{1,2^{*}}$, ZHI DAI $^{1,2^{*}}$, KANG SONG $^{1,2^{*}}$, ZHUO-JUN ZHANG $^{2 *}$, ZHENG-JUN ZHOU ${ }^{1,2}$, SHAO-LAI ZHOU ${ }^{1,2}$, \\ YI-MING ZHAO ${ }^{1,2}$, YONG-SHENG XIAO ${ }^{1}$, QI-MAN SUN ${ }^{1}$, ZHEN-BIN DING $^{1}$ and JIA FAN YH $^{1-3}$ \\ ${ }^{1}$ Liver Cancer Institute, Zhongshan Hospital, Key Laboratory of Carcinogenesis and Cancer Invasion, \\ Fudan University, Ministry of Education, Shanghai 200032; ${ }^{2}$ Shanghai Key Laboratory of Organ Transplantation, \\ Zhongshan Hospital, Fudan University, Shanghai; ${ }^{3}$ Institutes of Biomedical Sciences, Fudan University, Shanghai, P.R. China
}

Received August 21, 2014; Accepted October 2, 2014

DOI: 10.3892/ijo.2014.2761

\begin{abstract}
Macrophages are a major component of the leukocyte infiltrate of tumors and play a pivotal role in the progression of hepatocellular carcinoma (HCC). However, the molecular mechanisms by which macrophages promote HCC invasion are poorly understood. The present study was undertaken to investigate the relationship between macrophages and epithelial-mesenchymal transition (EMT) of HCC. Double-staining immunohistochemistry was used to observe the association between macrophages and EMT markers in clinical HCC samples and it showed that EMT primarily occurred at the edge of the tumor nest, in which infiltrating macrophages were always observed. This indicated that CD68 which is a marker of macrophages, was correlated with EMT marker levels. In addition, after being cultured with macrophages for $24 \mathrm{~h}$, the ability of HCC cells to migrate and invade increased, Snail and N-Cadherin expression was upregulated, and E-Cadherin was downregulated. An antibody array assay was applied to analyze the supernatant of these cultures and it demonstrated IL-8 increased significantly in the macrophage co-culture system. Finally, the role of macrophage-derived IL-8 in the invasion of HCC cells was assayed, and downstream signaling pathways were also investigated. We found that IL-8: i) may induce EMT and promote HCC cell migration and invasion and ii) is associated with the JAK2/STAT3/Snail signaling pathway. Taking together, these findings revealed that macrophages that have infiltrated tumors may induce
\end{abstract}

Correspondence to: Dr Jia Fan, Liver Cancer Institute, Zhongshan Hospital, Key Laboratory of Carcinogenesis and Cancer Invasion, Fudan University, Ministry of Education, 180 Fenglin Road, Shanghai 200032, P.R. China

E-mail: jiafan99@yahoo.com

*Contributed equally

Key words: hepatocellular carcinoma, CD68, IL-8, epithelialmesenchymal transition, STAT3 epithelial-mesenchymal transition of HCC cells via the IL-8 activated JAK2/STAT3/Snail pathway. Thus, this may offer a potential target for developing new HCC therapies.

\section{Introduction}

Hepatocellular carcinoma (HCC) is one of the most common types of malignant tumors worldwide, with high prevalence in Asia and South Africa (1). Surgical resection is the most common form of therapy (2), but HCC prognosis remains poor due to tumor invasiveness, frequent intrahepatic spread and extrahepatic metastasis (3).

Tumor metastasis is a complex process, and various factors are involved in each step of the process (4). Recent studies suggest that numerous genes and proteins play vital roles in the metastasis of cancer cells. Epithelial-mesenchymal transition (EMT) is the conversion of epithelial cells to mesenchymal cells; it is critical in the development of human cancer invasiveness and metastatic potential (5). During the transition process, intracellular adhesion between epithelial cells decreases and they acquire fibroblastoid properties, including downregulation of E-Cadherin, an epithelial marker, and upregulation of both N-Cadherin, a mesenchymal marker and Snail, which regulates EMT (6). EMT assists cancer cells in entering surrounding tissues by conferring invasive properties and allowing them to establish a suitable microenvironment for progression and metastasis (7). Iwatsuki et al (8) report that tumor buds, which involve a single cancer cell or small cluster of cells at the invasive front of tumor tissue, probably undergo EMT due to down-expression of E-Cadherin.

It has become clear that inflammatory cells present in the tumor microenvironment play an indispensable role in cancer progression (9). Tumor-associated macrophages, characterized as immunosuppressive, pro-angiogenic, and tumor growthpromoting, are a major component of the inflammatory cell infiltrate of tumors (10). In a previous study, we found that high levels of macrophage density, especially at the tumor edge, and peritumoral macrophage colony-stimulating factor (M-CSF) predicted high risk of recurrence and metastasis in HCC patients who had received curative liver resections (11). 
Jensen et al (12) also found that dense infiltration of $\mathrm{CD}^{+} 8^{+}$ macrophages at the tumor border were associated with poor survival in patients with melanoma. Chen et al (13)reported that macrophages can enhance the invasiveness and matrixdegrading activity of small cell lung cancer cell lines in vitro. Nevertheless, whether and how tumor-associated macrophages function in HCC progression and the relationship between tumor-infiltrating macrophages and EMT in HCC cells remain poorly understood.

By culturing HCC cell lines with macrophages, we were able to investigate the effect of the latter upon: i) HCC cell migration and invasion in vitro and ii) EMT in HCC cells. In addition, antibody microarrays were used to identify the cytokines mediating the association between paratumoral macrophages and EMT. To observe the relationship between macrophages and EMT in vivo, double-staining immunohistochemistry was used to detect macrophage density and EMT biomarker expression in specimens obtained from HCC patients.

\section{Materials and methods}

Cell preparations. MHCC97L and $\mathrm{MHCC} 97 \mathrm{H}$ are hepatitis $\mathrm{B}$ virus (HBV)-positive HCC cell lines with the same genetic background but different lung metastatic potential. Both were established at our institute. The human monocyte leukemia cell line THP-1 and two additional HCC cell lines, HepG2 and Huh7, which are HBV-negative cell lines with low metastatic potential, were purchased from the Institute of Biochemistry and Cell Biology, Chinese Academy of Sciences, Shanghai, China. MHCC97L, MHCC97H, HepG2 and Huh7 were cultured in Dulbecco's modified Eagle's medium (Invitrogen, Carlsbad, CA, USA) and THP-1 cells were cultured in RPMI-1640 medium (Invitrogen). Media were supplemented with $10 \%$ heat-inactivated fetal bovine serum (FBS) and $1 \%$ penicillin/streptomycin solution (Invitrogen). We cultured the cells at $37^{\circ} \mathrm{C}$ in a humidified atmosphere of $5 \% \mathrm{CO}_{2}$.

To obtain PMA-treated macrophages, we seeded $1 \times 10^{6}$ THP-1 cells into the upper insert of a 6-well Transwell apparatus (0.4 $\mathrm{mM}$ pore size; Corning, Lowell, MA, USA) and treated them with PMA $(320 \mathrm{nM})$ for $24 \mathrm{~h}$.

Patients and specimens. Clinical samples were collected after obtaining informed consent, according to an established protocol approved by the Ethics Committee of Fudan University. We collected information on HCC patients who received curative resections between January 2008 and December 2009 at the Liver Cancer Institute, Zhongshan Hospital, Fudan University, Shanghai, China. All data collected was de-identified. All patients included in the study underwent complete macroscopic removal of their tumors and did not have distant metastases or any prior anticancer treatment. The pathologic features of all cases were reviewed by an experienced pathologist blinded to the original pathology reports. Patients were divided into two groups, according to whether HCC had recurred within two years. By using random numbers generated via SPSS software version 16 (SPSS, Inc., Chicago, IL, USA), 20 patients from each group were selected for double staining immunohistochemistry. In total, 40 cases were picked, and 40 paraffin-embedded samples were obtained, including tumoral and paratumoral tissue, especially from the tumor edge.

Establishment of co-culture system with macrophages and HCC cells. After a thorough wash, PMA-treated THP-1 macrophages (upper inserts) were co-cultured with HCC cells (in a 6 -well plate, $2 \times 10^{5}$ cells/well) without direct contact. After $24 \mathrm{~h}$ of co-culture, the upper inserts containing the macrophages were discarded, and HCC cells were washed and used for subsequent experiments.

Cell migration and Matrigel invasion assays: co-cultured HCC cells. A wound-healing assay was used to evaluate the ability of HCC cells to migrate following culture with PMA-treated macrophages. Cells were grown to $80-90 \%$ confluence in 24-well plates, and a wound was made by dragging a plastic pipette tip across the cell surface. The remaining cells were washed three times to remove cellular debris and incubated at $37^{\circ} \mathrm{C}$ with serum-free medium. Migrating cells at the wound front were photographed after $24 \mathrm{~h}$. All experiments were performed in triplicate.

Cell invasion assays were performed using 24-well Trans-wells $(8 \mu \mathrm{m}$ pore size; Corning) pre-coated with Matrigel (Falcon 354480; BD Biosciences, Franklin Lakes, NJ, USA). In total, $1 \times 10^{5}$ cells were suspended in $500 \mu 1$ DMEM containing 1\% FBS and added to the upper chamber, while $750 \mu 1$ DMEM containing $10 \%$ FBS was placed in the lower chamber. After $48 \mathrm{~h}$ of incubation, Matrigel and the cells remaining in the upper chamber were removed using cotton swabs. Cells on the lower surface of the membrane were fixed in $4 \%$ paraformaldehyde and stained with Giemsa. Cells in 5 microscopic fields (at x200 magnification) were counted and photographed. All experiments were performed in triplicate.

Double-staining immunohistochemistry: CD68, Snail and $\mathrm{N}$-Cadherin in HCC clinical specimens. The double immunohistochemical staining system is designed for the simultaneous detection of two antigens on one slide. Five- $\mu \mathrm{m}$ sections of paraffin-embedded clinical HCC samples were cut using a rotation microtome (Leica RM2125RT). The sections were deparaffinized in xylene $(2 \times 5 \mathrm{~min})$ and rehydrated in graded alcohols (100-70\%, 5 min each) and distilled water. After antigen retrieval with $0.01 \%$ EDTA pH 8.0 (boiled $10 \mathrm{~min}$ in a microwave), slides were processed with the Dako EnVision $^{\mathrm{TM}}$ Gl2 Double-stain System (K5361; Dako, Glostrup, Denmark). The primary antibodies were mouse anti-human CD68 (ready to use; IS61330; Dako), rabbit anti-human Snail (1:200; BS1853; Bioworld, Visalia, CA, USA), and rabbit antihuman N-Cadherin (1:1,000; ab18203; Abcam, Burlingame, CA, USA). The CD68 antibody was applied along with Snail and $\mathrm{N}$-Cadherin antibodies, respectively, to each specimen. Negative controls were treated identically except for the omission of the primary antibodies. Finally, the slides were counterstained with hematoxylin for 2 min then mounted with water-based mounting medium. Cells that stained positive for Snail and N-Cadherin were visualized using $\mathrm{DAB}^{+}$ Chromogen, and CD68 was visualized using permanent red chromogen. Macrophages were identified by red stain, while HCC cells undergoing EMT were identified by brown stain. 
A

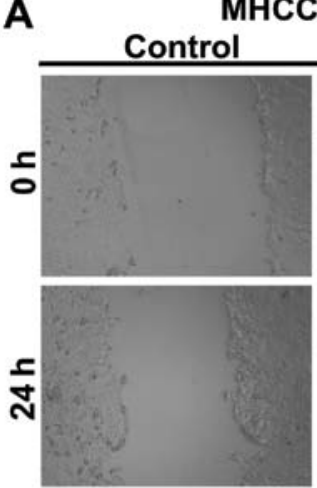

MHCC-97H

Co-cultured
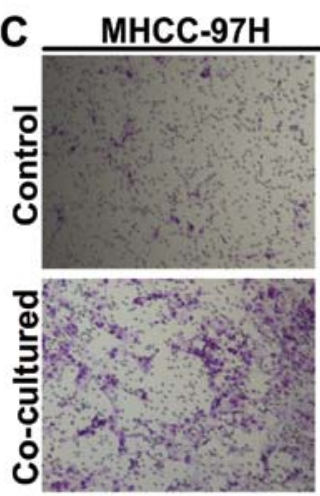
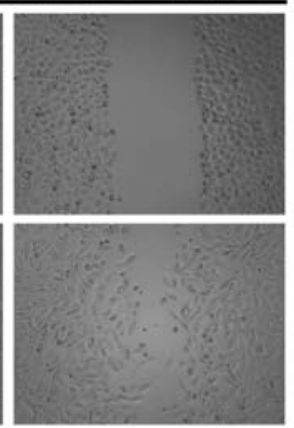

Hep-G2

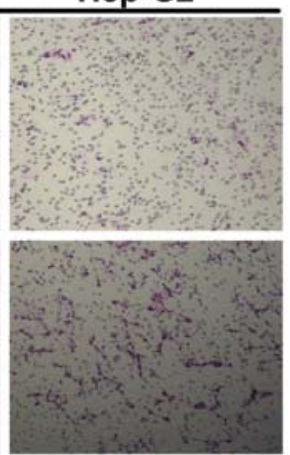

B Hep-G2
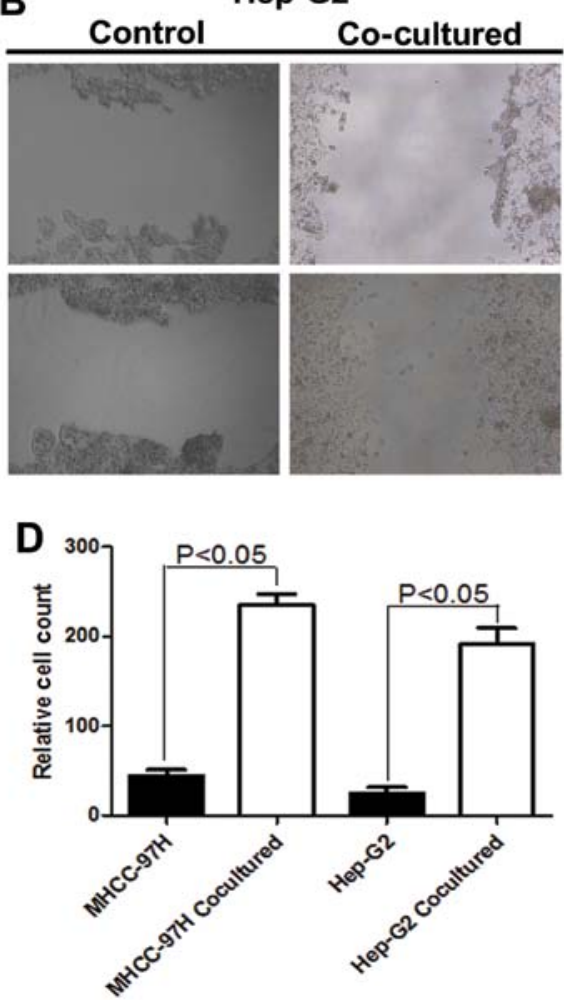

Figure 1. Macrophages enhance HCC cell migration and invasion in vitro. Functional analysis is illustrated after co-cultured with THP-1 macropahges in human hepatocellular cells in vitro. (A and B). After co-cultured with THP-1 macrophages, MHCC-97H and Hep-G2 migrated rapidly compared with control group for wound channel closure (original magnification, x100). (C and D) A Transwell assay showed that the number of invading HCC cells were higher than in control groups (original magnification, x100).

In each section, staining was captured by Leica QWin Plus version 3 software (Leica Microsystems, Wetzlar, Germany).

Western blotting: EMT markers and the JAK2/STAT3 pathway in HCC co-cultures. Protein $(30 \mu \mathrm{g})$ from the total cell extract of cell cultures was used to perform sodium dodecyl sulfatepolyacrylamide gel electrophoresis (SDS-PAGE), then proteins were transferred onto polyvinylidene difluoride membranes and incubated with the corresponding antibodies. The membranes were developed using the enhanced chemiluminescence method (Pierce, Rockford, IL, USA). Rabbit anti-human Snail polyclonal antibody (1:1,000; BS1853; Bioworld), rabbit antihuman E-Cadherin polyclonal antibody (1:10,000; ab40772; Abcam), and rabbit anti-human N-Cadherin polyclonal antibody (1:1,000; ab18203; Abcam) were used to detect the expression of Snail, E-Cadherin and N-Cadherin, respectively. Rabbit anti-human JAK2/p-JAK2 monoclonal antibody (1:1,000; 3230; Cell Signaling Technology, Danvers, MA, USA/1:1,000; 4406; Cell Signaling Technology) and rabbit anti-human STAT3/p-STAT3 monoclonal antibody (1:1,000; 4904; Cell Signaling Technology/1:2,000; 9145; Cell Signaling Technology) were selected to detect the JAK2/STAT3 pathway. A monoclonal $\beta$-tubulin antibody (1:1,000; Beyotime Institute of Biotechnology, Haimen, China) was used as an internal control. Horseradish peroxidase-conjugated anti-mouse and anti-rabbit antibody (1:5,000; KC-RB-035; KangCheng Biotechnology, Shanghai, China) were used as secondary antibodies. The intensity of protein bands was determined by densitometry using the Bio-Rad system (Bio-Rad Laboratories, Hercules, CA, USA). All experiments were performed in triplicate.

Immunofluorescence analysis: EMT markers in HCC co-cultures. Immunofluorescence analysis of tumor cells cultured on glass coverslips was performed as previously described (14). Cells were cultured for $72 \mathrm{~h}$ in the appropriate medium containing antibodies specific for E-Cadherin (1:500; ab40772; Abcam), N-Cadherin (1:50; ab18203; Abcam) and Snail (1:100; BS1853; Bioworld).

Antibody chip assay and ELISA verification of cytokines produced in HCC co-cultures. Samples of serum-free culture media were obtained from the supernatant of co-cultured HCC cells (MHCC-97H and Hep-G2) and macrophages. The levels of 80 human cytokines were determined using the human cytokine antibody array G-Series 5 (RayBiotech, Inc., Norcross, GA, USA). Briefly, a panel of antibodies is immobilized in specific locations on the surface of a glass slide. Incubating samples with the array results in the capture of cytokines by the corresponding antibodies. Bound cytokines are then detected with a cocktail of biotinylated antibodies. Signals are visualized using streptavidin-HRP conjugate and colorimetry. Results are expressed in relative units of spot color density, which reflect the concentration of each chemokine. Color images of the array were analyzed using the Image Lab 3.0. 


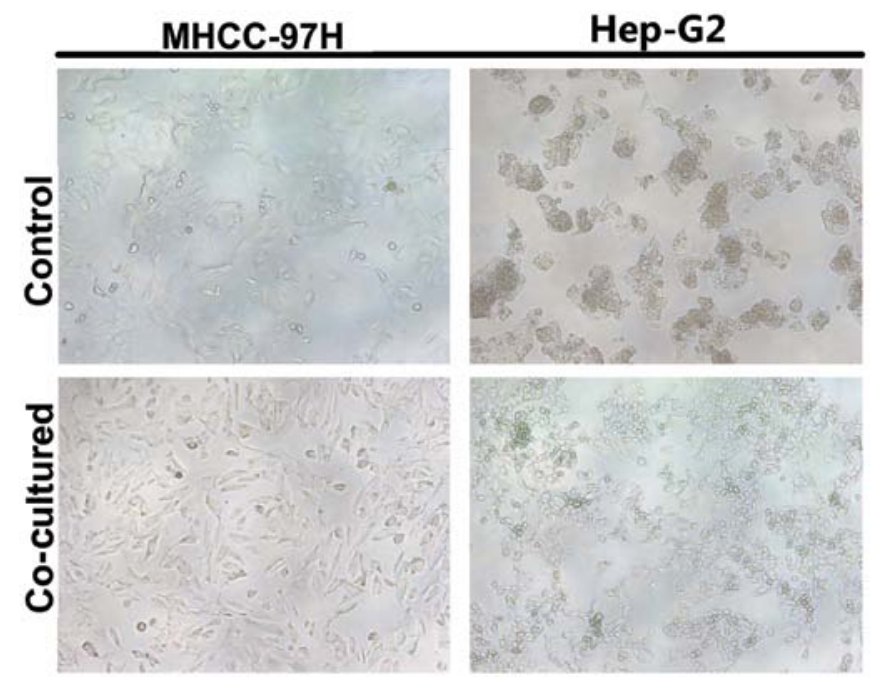

Figure 2. Morphological change of HCC cells. After being cultured with macrophages, HCC cells were scattered and spindle shaped, especially the Hep-G2 cell line; originally growing in a mass, Hep-G2 began to grow in a solitary fashion after macrophage co-culture. In the co-cultured HCC cells elongated parapodium could be observed (original magnification, x200).

Levels of MIP-3 $\alpha$, TNF- $\alpha$, RANTES, MCP-1, IL-6, IL-8, IL- $1 \beta$ and GRO- $\alpha$ were measured via ELISA in serum-free supernatant from HCC cell cultures, following the manufacturer's instructions (R\&D Systems, Inc., Minneapolis, MN, USA).

Statistical analysis. Statistical analysis was performed using SPSS 16.0 software. Values are expressed as means \pm standard deviations. The normality of the data was assessed by the
Kolmogorov-Smirnov test. For normally distributed variables, the experimental and control groups were compared using the Student's t-test. The cut-off for statistical significance was $\mathrm{P} \leq 0.05$.

\section{Results}

Macrophages enhance HCC cell migration and invasion in vitro. Phorbol myristyl acetate (PMA)-treated THP-1 macrophages were co-cultured with HCC cells in a non-contact Transwell system. Matrigel invasion assays showed that the number of invading HCC cells increased significantly after being co-cultured with macrophages (Fig. 1C) (MHCC-97H, $44.7 \pm 6.5$ vs. $234.7 \pm 13.3$; Hep-G2, $25.0 \pm 6.0$ vs. $191.7 \pm 17.6$, $\mathrm{P}<0.05$; Fig. 1D). In addition, a wound-healing assay, used to measure migration, demonstrated accelerated wound closure in HCC cells cultured with macrophages (Fig. 1A and B).

Culturing HCC cells with macrophages is associated with EMT. Control HCC cells formed clusters in culture and had tight cellular junctions. By contrast, after being cultured with macrophages, HCC cells were scattered and spindle shaped, similar to fibroblast cells, especially the Hep-G2 cell line; originally growing in a mass, Hep-G2 began to grow in a solitary fashion after macrophage co-culture. In the co-cultured HCC cells elongated parapodium could be observed (Fig. 2). To clarify whether EMT had taken place, we assessed the expression of Snail, E-Cadherin, and N-Cadherin, three key markers of the EMT process, in $\mathrm{HCC} /$ macrophage co-cultures using western blots. The results showed that Snail and $\mathrm{N}$-Cadherin expression was significantly enhanced in HCC cells co-cultured with macrophages, while E-Cadherin expression decreased sharply (Fig. 3).
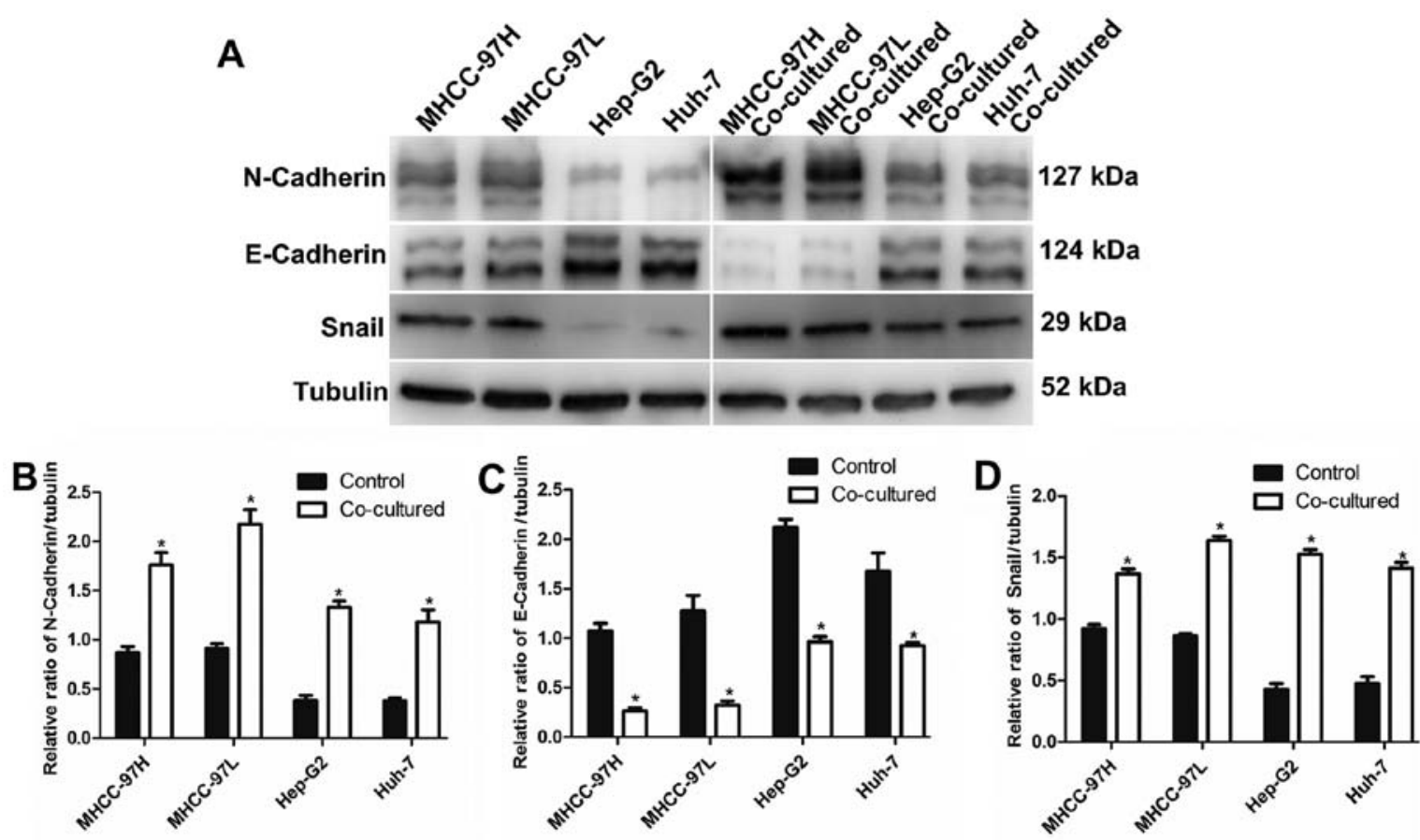

Figure 3. Culturing HCC cells with macrophages is associated with EMT. The expression of Snail, E-Cadherin and N-Cadherin, three key markers of the EMT process were assessed. (A) Western blots analysis demonstrated the expression of Snail, N-Cadherin and E-Cadherin in HCC cell lines and the ones co-cultured with macrophages. (B-D) Semi-quantitative analysis demonstrated that Snail and N-Cadherin expression was significantly enhanced in HCC cells co-cultured with macrophages, while E-Cadherin expression decreased sharply ( $\mathrm{P}<0.05)$. 

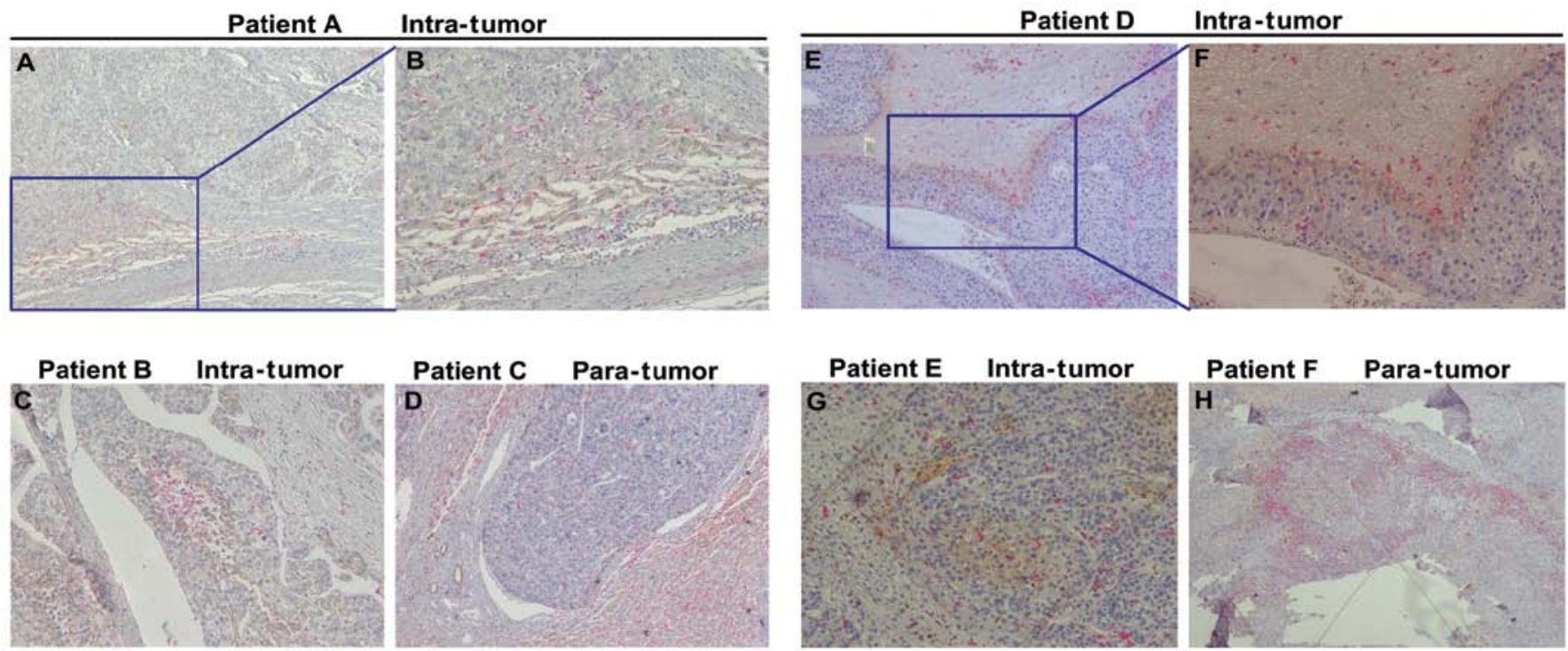

Figure 4. EMT in clinical HCC specimens. The expression of CD68, Snail and N-Cadhrin was detected by double-staining immunohistochemistry analysis (A-D) CD68 (red) and N-Cadherin (brown) were stained simultaneously on one slide. CD68-positive macrophages mainly infiltrated the stroma of the tumor, and N-Cadherin-positive tumor cells were surrounded by macrophages. (E-H) CD68 (red) and Snail (brown) were stained simultaneously on one slide. Macrophages were mainly observed around the edge of the tumor nest and in the area where macrophages were concentrated. The expression of Snail was also stronger in such areas. (A, C, D and E, original magnification, x100; B, F and G, original magnification, x200; H, original magnification, x40).

Table I. Concentration of cytokines in the cell culture medium.

\begin{tabular}{|c|c|c|c|c|}
\hline & \multicolumn{2}{|c|}{ MHCC-97H } & \multicolumn{2}{|c|}{ Hep-G2 } \\
\hline & Control & Co-cultured & Control & Co-cultured \\
\hline MIP-3 $\alpha$ & $54.65 \pm 8.87$ & $57.42 \pm 12.67$ & $58.99 \pm 6.71$ & $68.41 \pm 11.48$ \\
\hline TNF- $\alpha$ & $28.95 \pm 6.03$ & $603.89 \pm 15.18$ & $30.86 \pm 4.18$ & $625.32 \pm 56.43$ \\
\hline RANTES & $31.21 \pm 4.14$ & $36.21 \pm 3.96$ & $20.34 \pm 3.14$ & $22.89 \pm 8.48$ \\
\hline MCP-1 & $442.38 \pm 20.95$ & $451.20 \pm 34.66$ & $527.00 \pm 77.71$ & $571.55 \pm 92.29$ \\
\hline IL-6 & $17.76 \pm 3.55$ & $911.68 \pm 67.24$ & $27.27 \pm 9.27$ & $848.32 \pm 57.29$ \\
\hline IL-8 & $16.74 \pm 2.09$ & $4317.74 \pm 291.08$ & $29.01 \pm 14.13$ & $4347.40 \pm 192.09$ \\
\hline IL-1 $\beta$ & $13.57 \pm 2.05$ & $461.42 \pm 26.73$ & $28.69 \pm 5.45$ & $525.36 \pm 34.553$ \\
\hline GRO- $\alpha$ & $15.03 \pm 2.48$ & $15.24 \pm 5.57$ & $12.95 \pm 2.38$ & $14.01 \pm 2.04$ \\
\hline
\end{tabular}

EMT in clinical HCC specimens. Double-staining immunohistochemistry analysis of HCC clinical samples showed that macrophages which stained CD68 positive were mainly observed around the edge of the tumor nest (Fig. 4). Notably, in the area where macrophages were concentrated, Snail and $\mathrm{N}$-Cadherin expression was high.

Macrophage-derived IL-8 induces EMT in cultured HCC cells. We used antibody arrays to investigate which macrophage-secreted cytokines promoted HCC cell invasion in our co-culture model. Serum-free culture media was obtained from cell-normalized cultures; as shown in Fig. 5, levels of macrophage inflammatory protein-3 $\alpha$ (MIP-3 $\alpha$ ), tumor necrosis factor- $\alpha$ (TNF- $\alpha$ ), RANTES, monocyte chemoattractant protein (MCP-1), IL-6, IL-8, IL-1 $\beta$ and growth related oncogene- $\alpha(\mathrm{GRO}-\alpha)$ were significantly elevated in both co-cultured MHCC-97H and Hep-G2 cells.
In order to verify the results obtained from the array, ELISA was used to assess levels of the cytokines listed above in the supernatant of $\mathrm{HCC} /$ macrophage co-cultures and HCC control cultures. No significant differences between MIP-3 $\alpha$, RANTES, MCP-1 and GRO- $\alpha$ levels were found between the co-cultured and control groups, but the levels of TNF- $\alpha$, IL-6, IL- 8 and IL-1 $\beta$ increased significantly in the media from co-cultured HCC cells (Table I; Fig. 6A and B). As shown in Fig. 6C and D, we observed a nearly 100 -fold increase in levels of secreted IL- 8 in the supernatant of HCC cells cultured with macrophages relative to control HCC cells.

IL-8 induces a mesenchymal-like phenotype in HCC cells via dose-related activation of the JAK2/STAT3 pathway. To investigate whether IL-8 and JAK2/STAT3 activation plays a critical role in inducing EMT, we cultured Hep-G2 cells with macrophages in the presence of varying levels of IL- $8(0,1$, 

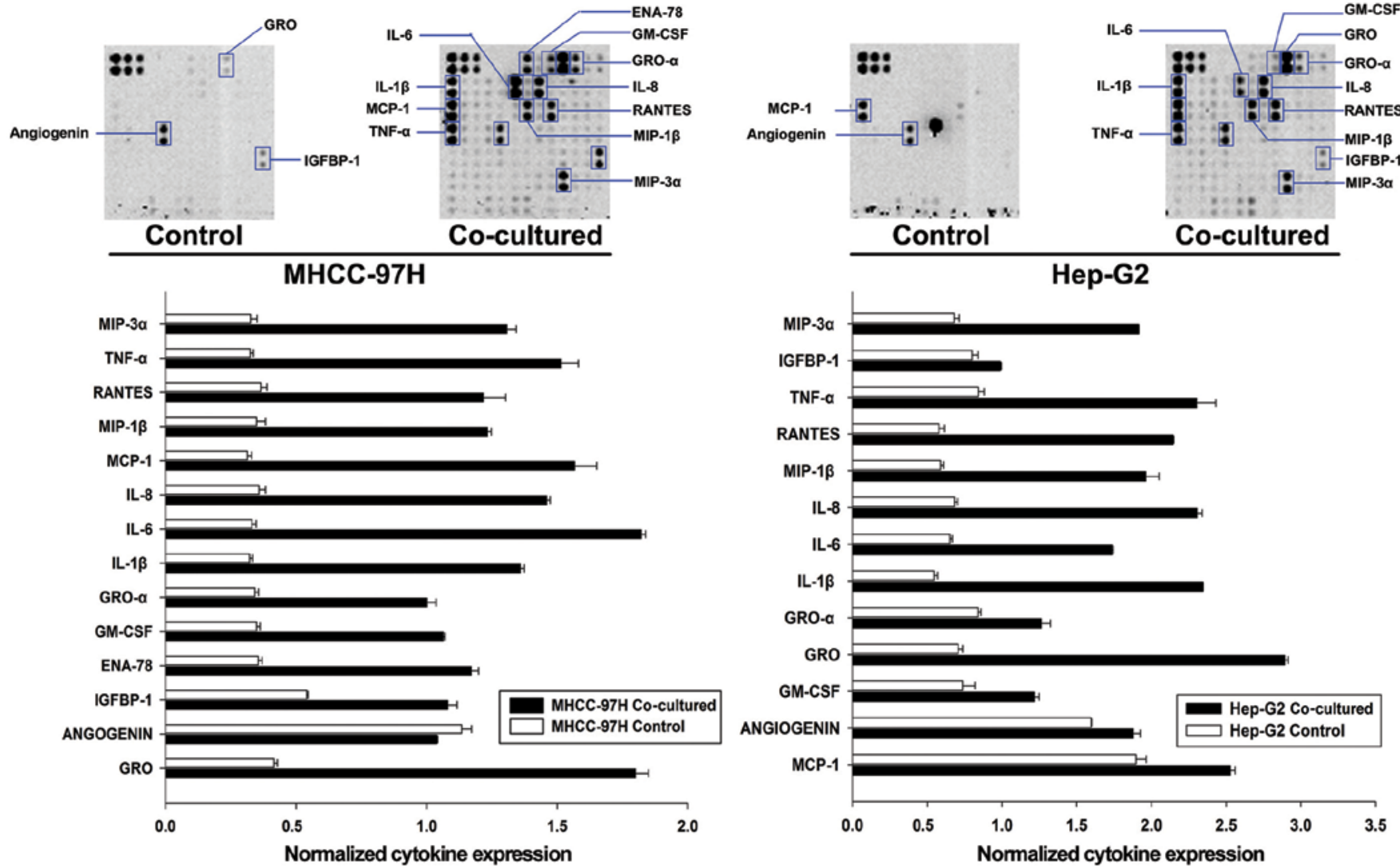

Figure 5. Antibody arrays. Serum-free culture supernatants from MHCC-97H/Hep-G2 and macrophage-MHCC-97H/Hep-G2 co-culture system were analyzed by antibody arrays. Shown in the graphs is the expression of soluble factors that expressed a significant change.
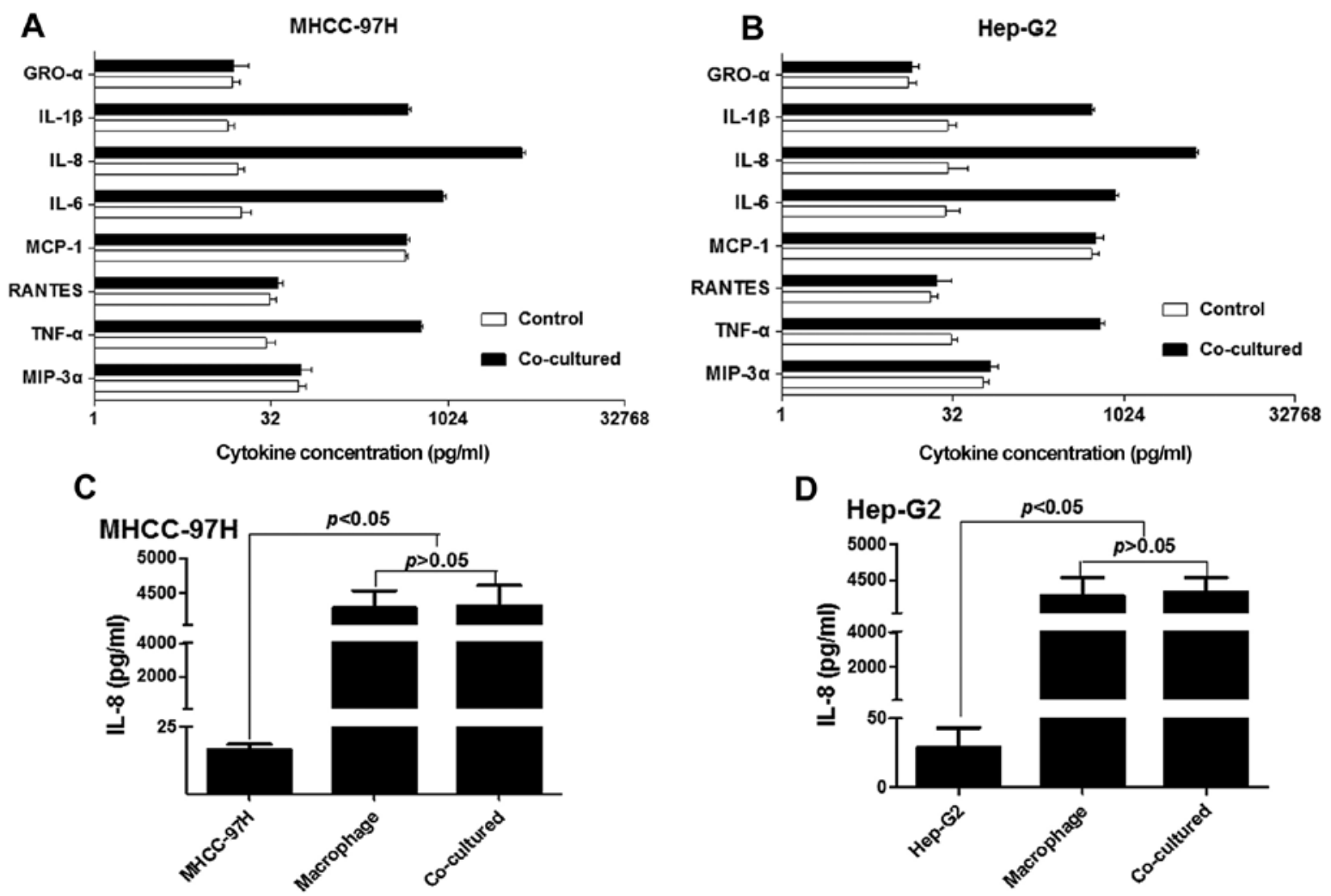

Figure 6. ELISA verification of cytokines produced in HCC co-cultures (A and B). The cytokines that had been discovered by antibody arrays (in Fig. 5) were verified by ELISA, and the supernatants of MHCC-97H/Hep-G2 and macrophage-MHCC-97H/Hep-G2 co-culture systems were obtained for analysis. (C and D) The IL-8 levels in the supernatant of MHCC-97H, Hep-G2, macrophages and macrophage-MHCC-97H/Hep-G2 co-culture systems were analyzed by ELISA. 
A

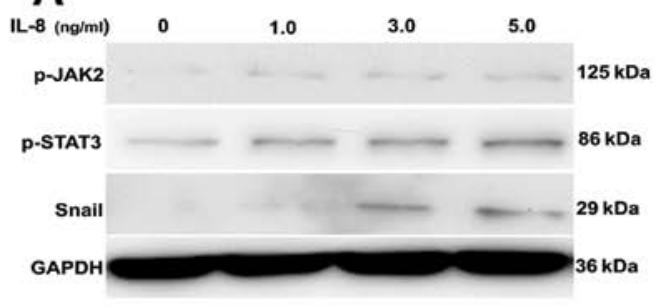

C

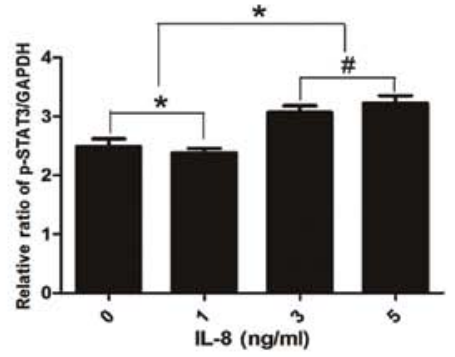

B

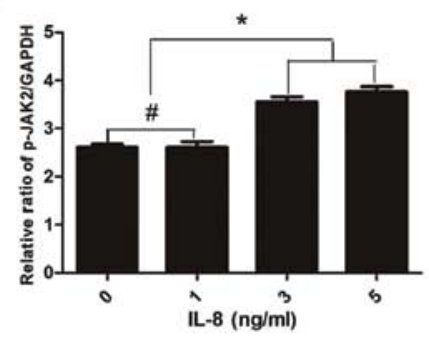

D

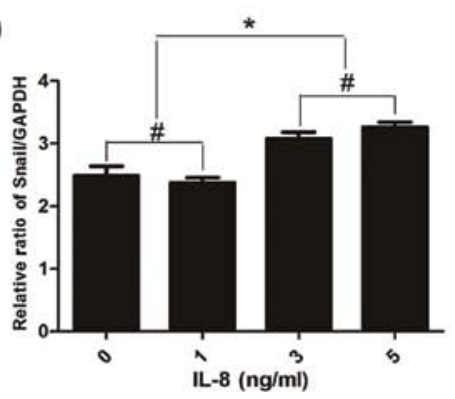

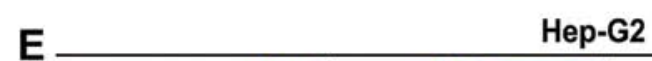

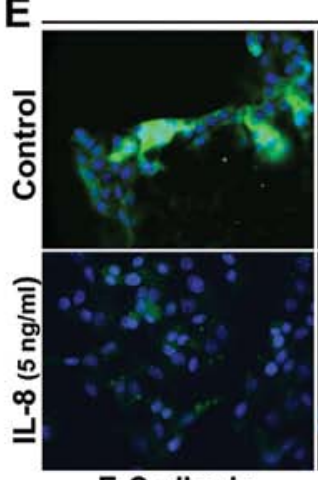

E-Cadherin

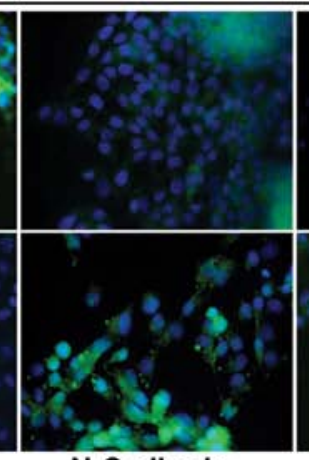

N-Cadherin

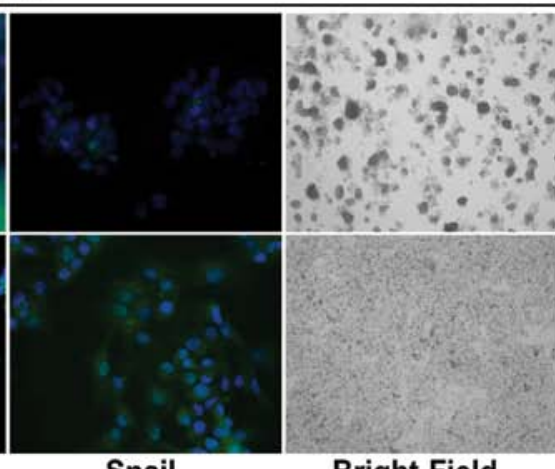

Bright-Field

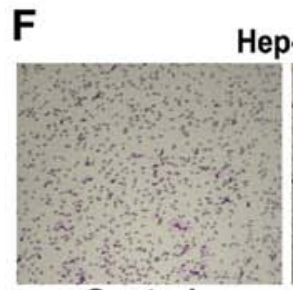

Control
Hep-G2

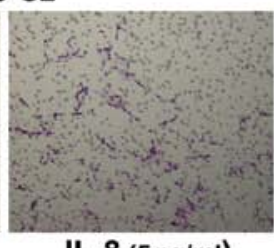

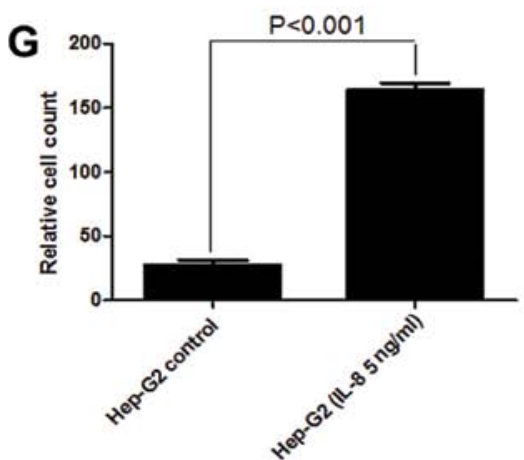

Figure 7. Role of IL-8 signaling in EMT. (A) The expression of Jak2, p-Jak2, Stat3, p-Stat3 and Snail were assessed in Hep-G2 exposure to different concentrations of IL-8. (B-D) Semi-quantitative analysis demonstrated that Jak2/Stat3/Snail pathway could be activated by IL-8. $\left({ }^{*} \mathrm{P}>0.05\right.$; $\left.{ }^{*} \mathrm{P}<0.05\right)$. (E) Immunofluorescent analysis of E-Cadherin, N-Cadherin and Snail in Hep-G2 vs. Hep-G2 that was incubated with IL-8. The green signal represents the staining of the corresponding protein and the blue signal represents the DAPI-stained nuclei. The morphological change of Hep-G2 after incubated with IL-8 was also observed. (F and G) Functional analysis is illustrated after IL-8 had been added to the media for $24 \mathrm{~h}$. A Transwell assay showed that the number of invading Hep-G2 cells were higher that in control groups (original magnification, x100).

3 and $5 \mathrm{ng} / \mathrm{ml}$ ) for $24 \mathrm{~h}$, subsequently testing their invasive potential in vitro. JAK2/STAT3 activation was indicated by an increase in phosphor-specific forms of JAK2/STAT3 relative to total forms of JAK2/STAT3. We found the activity of both JAK2 and STAT3 increased with increasing IL-8 concentrations (Fig. 7A-D).
In addition, we observed that the ability of Hep-G2 cells to invade the extracellular matrix in vitro increased in the presence of IL-8 (Fig. 7F and G). The shape of the cells became fusiform and the conjunctions between them loose. Immunofluorescence analysis also revealed that levels of the epithelial marker, E-Cadherin, decreased, while levels of 
the mesenchymal markers, N-Cadherin and Snail, increased (Fig. 7E).

\section{Discussion}

Multiple studies on various types of human carcinomas have demonstrated a positive association between the activation of an EMT program and poor prognosis or advanced disease (15-18). This suggests that EMT plays a critical role in cancer progression. The tumor microenvironment is composed of an extracellular matrix, fibroblasts, myofibroblasts, endothelial cells, immune cells and soluble factors. A recent study revealed that stromal cells in solid tumors represent a dynamic, flexible asset for tumor progression (19), and it is recognized that the balance between tumor immunity and tumor progression is important (20). Macrophages, an important component of the tumor microenvironment, was observed mainly to infiltrate the stromal area and it is reported that they can promote tumor progression and are associated with poor prognosis (21-25). By using doublestaining immunohistochemistry to investigate clinical samples, we found that the number of HCC cells that switch from an epithelial to a mesenchymal-like phenotype may constitute only a small fraction of the total primary tumor mass; mesenchymal-like cells were especially common at the edge of tumor nests, while macrophages were primarily observed in the border areas of the tumor nest and stroma. This phenomenon indicates that macrophages that have infiltrated the tumor stroma may play a key role in the EMT process in HCC. The double-staining immunohistochemistry was applied only to observe the location of macrophages and tumor cells which were undergoing EMT in HCC tissues, and no statistical analyses were performed.

As mentioned above, macrophages were part of tumor's adjacent stroma, and their correlations with patient survival have largely been related to the macrophage secretome which involves factors that stimulate tumor cell proliferation and survival, angiogenesis and release of proteases essential for extracellular matrix remodeling (26-28). In the present study, we hypothesized that macrophages that infiltrate the stroma could influence tumor cells in the epithelial state by secretion of certain cytokines. In order to determine whether the associations are causal, we used a non-contact system described previously for co-culture macrophages and HCC cells and THP-1 macrophages were used as macrophage model because PMA-treated THP-1 macrophages had an M2 functional profile $(13,29)$. We demonstrated that culturing two HCC cell lines with macrophages led to EMT, upexpression of two mesenchymal markers, N-Cadherin and Snail, and induction of invasiveness. Therefore, it is possible that soluble factors secreted by macrophages could induce adjacent epithelial tumor cells to undergo EMT and acquire metastatic potential.

Although our antibody array results indicated that the transition of HCC cells from an epithelial to a mesenchymal-like phenotype was associated with the secretion of multiple cytokines and chemokines, including MIP- $3 \alpha$, TNF- $\alpha$, RANTES, MCP-1, IL-6, IL-8, IL-1 $\beta$ and GRO- $\alpha$, when ELISA was used to verify these differences, only levels of TNF- $\alpha$, IL-6, IL- 8 and IL- $1 \beta$ were found to increase. IL- $1 \beta$ and TNF- $\alpha$ are reported to regulate IL-8 expression in fibroblasts (30), endothelial cells (31), gastric carcinoma (32) and prostate cancer (33). Soria et al (34) found that coordinated expression of TNF- $\alpha$ and IL-1 $\beta$ may promote breast cancer recurrence. Moreover, Akiba et al (35) discovered that cancerous tissue samples with high IL-8 levels have a significantly higher frequency of portal vein invasion. Various studies have also shown that IL-6 is important in the pathogenesis of HCC, and it has been known for some time that, in humans, IL-6 levels are increased in serum from patients with chronic liver disease, including cirrhosis and HCC $(36,37)$. Recent studies have indicated that IL- 6 is capable of inducing EMT in human breast cancer cells (38), so we cannot reject the possibility that IL-6 contributes to EMT in HCC.

IL-8 expression in cancer has been associated with tumor growth and survival, increased tumor cell migration and invasion, and increased neovascularization (39-41). Therefore, IL-8 was the major focus of the present study. In this study, IL-8 levels increased in cultures containing both HCC cells and macrophages, and this increase was much greater than that seen for IL-6. We hypothesize that the large increase in IL- 8 levels was due to synergy between TNF- $\alpha$ and IL-1 $\beta$. Our ELISA results showed that IL-8 concentrations were mainly elevated in the supernatant of cultured macrophages and co-cultured HCC cells and macrophages, while the levels of IL-8 in cultured HCC cells were appreciably lower. This suggests that IL- 8 is secreted primarily by the macrophages and is probably involved in the development of HCC in vitro.

STAT3 is regarded as a critical transcription activator for cell cycle or cell survival-related genes, and its phosphorylation has been linked to HCC tumor progression (42), angiogenesis (43) and tumorigenesis (44). Colomiere et al (45) have reported that activation of the JAK2/STAT3 pathway may result in EMT-associated phenotypes of ovarian cancer cells. Moreover, consistent suppression of STAT3 activity may abrogate $\mathrm{N}$-Cadherin and vimentin expression, consistent with the loss of cell motility in ovarian cancer (46). In a study of head and neck tumors, the JAK2/STAT3/Snail signaling pathway was identified as the major factor in inducing EMT (47), but little attention was paid to the tumor stroma, which could be the most important trigger for EMT. Fernando et al (48) illustrated the essential role of IL-8 signaling in the acquisition and/or maintenance of the mesenchymal and invasive features of Brachyury-overexpressing tumor cells; they also showed that IL-8 secreted by HCC cells undergoing EMT could potentiate tumor progression by inducing adjacent epithelial tumor cells to undergo EMT (48). These authors, however, did not investigate pathways downstream of IL-8 that may induce EMT. Therefore, we investigated the hypothesis that HCC cells may undergo EMT via the JAK2/STAT3 pathway. Our western blot data from HCC cultures showed that JAK2/ STAT3 activation was positively associated with IL-8 levels in a dose-dependent fashion. Moreover, Snail expression also increased with increasing IL-8.

Our data provide novel evidence for the importance of macrophages in HCC pathogenesis. Moreover, we confirm that the soluble cytokines secreted by macrophages, such as IL-8, can trigger a switch in the phenotype of HCC cells. In light of these results, the development of strategies aimed at interfering with cytokines appears to be a rational approach for preventing metastasis, which would improve HCC thera- 
peutic efficacy. In addition, our results indicate that: i) IL-8 is the most probable candidate cytokine to induce EMT in HCC cells and ii) the JAK2/STAT3/Snail signaling pathway may lie downstream of the IL-8 receptor axis. Our research demonstrates, for the first time, the mechanism by which macrophages that have infiltrated the tumoral stroma can induce EMT in HCC. They also provide a new perspective on how IL-8 is linked to the JAK2/STAT3/Snail pathway.

There are several potential limitations of the present study. It should be noted that this study primarily focused on macrophages that infiltrated the stroma of the tumor. However, the tumor immune microenvironment is composed of various kinds of immune-related cells, such as neutrophils (49), natural killer T cells (50), T lymphocytes (51) and T regulatory cells (52), which can influence the biological activity of HCC cells. Although the macrophage is widely recognized as the major type of inflammatory cell that infiltrates tumors, we cannot be certain that the macrophage is the only cell type influencing EMT in HCC. In addition, in this study, we focused primarily on the effect of IL-8 rather than those of the other cytokines identified by our antibody array. As mentioned above, IL-6 was another potential trigger of EMT in HCC. Thus, future studies should investigate the role of additional cytokines in affecting HCC pathogenesis; moreover, an attempt to characterize the network of immune-related cytokines in HCC should be considered.

\section{Acknowledgements}

The present study was supported by the funds from the National Natural Science Fund of China (nos. 81272724 and 81472218), the Major Program of NSFC (no. 81030038), the National Key Sci-Tech Project, China (2012ZX10002011002 and 2012ZX10002013-005) and the Young Investigator Award of Zhongshan Hospital, Fudan University, China (no. 2013ZSQN16).

\section{References}

1. El-Serag HB and Rudolph KL: Hepatocellular carcinoma: epidemiology and molecular carcinogenesis. Gastroenterology 132 2557-2576, 2007.

2. Clavien PA, Petrowsky H, DeOliveira ML and Graf R: Strategies for safer liver surgery and partial liver transplantation. N Engl J Med 356: 1545-1559, 2007.

3. Huo TI, Lin HC, Huang YH, et al: The model for end-stage liver disease-based Japan Integrated Scoring system may have a better predictive ability for patients with hepatocellular carcinoma undergoing locoregional therapy. Cancer 107: 141-148, 2006

4. Nakakura EK and Choti MA: Management of hepatocellular carcinoma. Oncology (Williston Park) 14: 1085-1102, 2000.

5. Thiery JP: Epithelial-mesenchymal transitions in tumour progression. Nat Rev Cancer 2: 442-454, 2002.

6. Yang J and Weinberg RA: Epithelial-mesenchymal transition: at the crossroads of development and tumor metastasis. Dev Cell 14: 818-829, 2008.

7. Yilmaz M and Christofori G: EMT, the cytoskeleton, and cancer cell invasion. Cancer Metastasis Rev 28: 15-33, 2009.

8. Iwatsuki M,Mimori K, Yokobori T, et al: Epithelial-mesenchymal transition in cancer development and its clinical significance. Cancer Sci 101: 293-299, 2010.

9. Budhu A, Forgues M, Ye QH, et al: Prediction of venous metastases, recurrence, and prognosis in hepatocellular carcinoma based on a unique immune response signature of the liver microenvironment. Cancer Cell 10: 99-111, 2006.
10. Sica A, Larghi P, Mancino A, et al: Macrophage polarization in tumour progression. Semin Cancer Biol 18: 349-355, 2008.

11. Zhu XD, Zhang JB, Zhuang PY, et al: High expression of macrophage colony-stimulating factor in peritumoral liver tissue is associated with poor survival after curative resection of hepatocellular carcinoma. J Clin Oncol 26: 2707-2716, 2008.

12. Jensen TO, Schmidt H, Moller HJ, et al: Macrophage markers in serum and tumor have prognostic impact in American Joint Committee on Cancer stage I/II melanoma. J Clin Oncol 27: 3330-3337, 2009.

13. Chen JJ, Lin YC, Yao PL, et al: Tumor-associated macrophages: the double-edged sword in cancer progression. J Clin Oncol 23: 953-964, 2005.

14. Ding ZB, Shi YH,Zhou J, et al: Liver-intestine cadherin predicts microvascular invasion and poor prognosis of hepatitis B viruspositive hepatocellular carcinoma. Cancer 115: 4753-4765, 2009.

15. Fernando RI, Litzinger M, Trono P, Hamilton DH, Schlom J and Palena C: The T-box transcription factor Brachyury promotes epithelial-mesenchymal transition in human tumor cells. J Clin Invest 120: 533-544, 2010.

16. Blanco MJ, Moreno-Bueno G, Sarrio D, et al: Correlation of Snail expression with histological grade and lymph node status in breast carcinomas. Oncogene 21: 3241-3246, 2002.

17. Kwok WK, Ling MT, Lee TW, et al: Up-regulation of TWIST in prostate cancer and its implication as a therapeutic target. Cancer Res 65: 5153-5162, 2005.

18. Shih JY, Tsai MF, Chang TH, et al: Transcription repressor slug promotes carcinoma invasion and predicts outcome of patients with lung adenocarcinoma. Clin Cancer Res 11: 8070-8078, 2005.

19. de Visser KE, Eichten A and Coussens LM: Paradoxical roles of the immune system during cancer development. Nat Rev Cancer 6: 24-37, 2006.

20. Korangy F, Hochst B, Manns MP and Greten TF: Immune responses in hepatocellular carcinoma. Dig Dis 28: 150-154, 2010.

21. Bingle L, Brown NJ and Lewis CE: The role of tumour-associated macrophages in tumour progression: implications for new anticancer therapies. J Pathol 196: 254-265, 2002.

22. Pollard JW: Tumour-educated macrophages promote tumour progression and metastasis. Nat Rev Cancer 4: 71-78, 2004.

23. Lewis CE and Pollard JW: Distinct role of macrophages in different tumor microenvironments. Cancer Res 66: 605-612, 2006.

24. Siveen KS and Kuttan G: Role of macrophages in tumour progression. Immunol Lett 123: 97-102, 2009.

25. Stein M, Keshav S, Harris N and Gordon S: Interleukin 4 potently enhances murine macrophage mannose receptor activity: a marker of alternative immunologic macrophage activation. J Exp Med 176: 287-292, 1992.

26. Lin EY, Li JF, Gnatovskiy L, et al: Macrophages regulate the angiogenic switch in a mouse model of breast cancer. Cancer Res 66: 11238-11246, 2006

27. Zeisberger SM, Odermatt B, Marty C, Zehnder-Fjallman AH, Ballmer-Hofer K and Schwendener RA: Clodronate-liposomemediated depletion of tumour-associated macrophages: a new and highly effective antiangiogenic therapy approach. Br J Cancer 95: 272-281, 2006.

28. Gocheva V, Wang HW, Gadea BB, et al: IL-4 induces cathepsin protease activity in tumor-associated macrophages to promote cancer growth and invasion. Genes Dev 24: 241-255, 2010.

29. Chen JJ, Yao PL, Yuan A, et al: Up-regulation of tumor interleukin-8 expression by infiltrating macrophages: its correlation with tumor angiogenesis and patient survival in non-small cell lung cancer. Clin Cancer Res 9: 729-737, 2003.

30. Strieter RM, Phan SH, Showell HJ, et al: Monokine-induced neutrophil chemotactic factor gene expression in human fibroblasts. J Biol Chem 264: 10621-10626, 1989.

31. Strieter RM, Kunkel SL, Showell HJ, et al: Endothelial cell gene expression of a neutrophil chemotactic factor by TNF-alpha, LPS, and IL-1 beta. Science 243: 1467-1469, 1989.

32. Kitadai Y, Haruma K, Sumii K, et al: Expression of interleukin-8 correlates with vascularity in human gastric carcinomas. Am J Pathol 152: 93-100, 1998.

33. Kooijman R, Himpe E, Potikanond S and Coppens A: Regulation of interleukin-8 expression in human prostate cancer cells by insulin-like growth factor-I and inflammatory cytokines. Growth Horm IGF Res 17: 383-391, 2007. 
34. Soria G, Ofri-Shahak M, Haas I, et al: Inflammatory mediators in breast cancer: coordinated expression of TNFalpha \& IL-1beta with CCL2 \& CCL5 and effects on epithelial-to-mesenchymal transition. BMC Cancer 11: 130, 2011.

35. Akiba J, Yano H, Ogasawara S, Higaki $\mathrm{K}$ and Kojiro $\mathrm{M}$ : Expression and function of interleukin-8 in human hepatocellular carcinoma. Int J Oncol 18: 257-264, 2001.

36. Trikha M, Corringham R, Klein B and Rossi JF: Targeted anti-interleukin- 6 monoclonal antibody therapy for cancer: a review of the rationale and clinical evidence. Clin Cancer Res 9 : 4653-4665, 2003.

37. Tilg H, Wilmer A, Vogel W, et al: Serum levels of cytokines in chronic liver diseases. Gastroenterology 103: 264-274, 1992.

38. Sullivan NJ, Sasser AK, Axel AE, et al: Interleukin-6 induces an epithelial-mesenchymal transition phenotype in human breast cancer cells. Oncogene 28: 2940-2947, 2009.

39. Park SH, Kim JH, Lee DH, et al: Luteolin 8-C-betafucopyranoside inhibits invasion and suppresses TPA-induced MMP-9 and IL-8 via ERK/AP-1 and ERK/NF-kappaB signaling in MCF-7 breast cancer cells. Biochimie 95: 2082-2090, 2013.

40. Lattanzio L, Tonissi F, Torta I, et al: Role of IL-8 induced angiogenesis in uveal melanoma. Invest New Drugs 31: 1107-1114, 2013.

41. Yuan A, Chen JJ, Yao PL and Yang PC: The role of interleukin-8 in cancer cells and microenvironment interaction. Front Biosci 10: 853-865, 2005.

42. Rajendran P, Ong TH, Chen L, et al: Suppression of signal transducer and activator of transcription 3 activation by butein inhibits growth of human hepatocellular carcinoma in vivo. Clin Cancer Res 17: 1425-1439, 2011.

43. Yang SF, Wang SN, Wu CF, et al: Altered p-STAT3 (tyr705) expression is associated with histological grading and intratumour microvessel density in hepatocellular carcinoma. J Clin Pathol 60: 642-648, 2007

44. Ogata H, Kobayashi T, Chinen T, et al: Deletion of the SOCS3 gene in liver parenchymal cells promotes hepatitis-induced hepatocarcinogenesis. Gastroenterology 131: 179-193, 2006.
45. Colomiere M, Ward AC, Riley C, et al: Cross talk of signals between EGFR and IL-6R through JAK2/STAT3 mediate epithelial-mesenchymal transition in ovarian carcinomas. Br J Cancer 100: 134-144, 2009.

46. Colomiere M, Findlay J, Ackland L and Ahmed N: Epidermal growth factor-induced ovarian carcinoma cell migration is associated with JAK2/STAT3 signals and changes in the abundance and localization of alpha6betal integrin. Int J Biochem Cell Biol 41: 1034-1045, 2009

47. Yadav A, Kumar B, Datta J, Teknos TN and Kumar P: IL-6 promotes head and neck tumor metastasis by inducing epithelialmesenchymal transition via the JAK-STAT3-SNAIL signaling pathway. Mol Cancer Res 9: 1658-1667, 2011.

48. Fernando RI, Castillo MD, Litzinger M, Hamilton DH and Palena C: IL-8 signaling plays a critical role in the epithelialmesenchymal transition of human carcinoma cells. Cancer Res 71: 5296-5306, 2011.

49. Zhou SL, Dai Z, Zhou ZJ, et al: Overexpression of CXCL5 mediates neutrophil infiltration and indicates poor prognosis for hepatocellular carcinoma. Hepatology 56: 2242-2254, 2012.

50. Xiao YS, Gao Q, Xu XN, et al: Combination of intratumoral invariant natural killer T cells and interferon-gamma is associated with prognosis of hepatocellular carcinoma after curative resection. PLoS One 8: e70345, 2013.

51. Xia YH, Wang ZM, Chen RX, et al: T-cell apoptosis induced by intratumoral activated hepatic stellate cells is associated with lung metastasis in hepatocellular carcinoma. Oncol Rep 30: 1175-1184, 2013

52. Yang P, Li QJ, Feng Y, et al: TGF-beta-miR-34a-CCL22 signaling-induced Treg cell recruitment promotes venous metastases of HBV-positive hepatocellular carcinoma. Cancer Cell 22: 291-303, 2012. 